\title{
Three-dimensional digital visible heart model and myocardial pathological characteristics of fetal single ventricle connected with aortic coarctation
}

\author{
B. Ren ${ }^{1 *}$, Y. Jiang ${ }^{1 *}$, H.M. Xia ${ }^{1}$, X.Y. Li ${ }^{1}$, L.W. Tan ${ }^{2}$, Y. Li ${ }^{2}$, Q.Y. Li ${ }^{2}$, \\ X.S. $\mathrm{Li}^{2}$ and Y.H. Gao ${ }^{1}$ \\ ${ }^{1}$ Department of Ultrasound, Second Affiliated Hospital, \\ Third Military Medical University, Chongqing, China \\ ${ }^{2}$ Department of Anatomy, Third Military Medical University, \\ Chongqing, China \\ *These authors contributed equally to this study. \\ Corresponding author: H.M. Xia \\ E-mail: bryjcn@163.com
}

Genet. Mol. Res. 12 (4): 5247-5256 (2013)

Received May 9, 2013

Accepted September 10, 2013

Published October 30, 2013

DOI http://dx.doi.org/10.4238/2013.October.30.9

\begin{abstract}
This study aimed to provide data for imaging diagnosis and clinical surgical plans by reconstructing a three-dimensional (3D) digital visible heart model of single ventricle (SV) connection with aortic coarctation $(\mathrm{CoA})$ and characterizing the myocardial and vascular wall pathological characteristics. Fifteen miscarried fetus cadavers with SV and CoA were selected. Fourteen cardiac specimens were systematically reviewed for segmental anatomy and conventional histological examinations. One fetus cadaver was used to obtain the structural dataset of the fetal body and to reconstruct a 3-D digital visible heart model. Specimen pathological dissection indicated hypertrophic myocardium SV, significant aortic wall thickening, and localized coarctation area elevation. Ten cases of SV with left ventricular morphology displayed a large muscle ridge and solitus
\end{abstract}


normally aligned great arteries. Five cases of SV with right ventricular morphology had coarse, parallel trabeculations and received a common atrioventricular valve. The reconstructed 3-D heart and the main internal structures were realistic, which were beneficial for clinical and image teaching of fetal heart development. The change of characteristics of the myocardium and great vascular wall was obvious and may be the critical cause leading to progressive dysfunction in the postnatal heart.

Key words: Digital visible heart model; Cardiomyopathy; Prenatal echocardiographic diagnosis

\section{INTRODUCTION}

In cardiology, obtaining precise information on the morphology and function of the heart is essential both for clinical applications (e.g., diagnosis, prognosis, therapeutic decisions) and for research fields. Single ventricle (SV) connection with aortic coarctation (CoA) is a group of abnormalities with aorta dysplasia and atrioventricular (AV) alignment consisting of a double inlet or common inlet ventricle in which there is only one ventricular sinus. There are two forms of SV: left ventricular morphology of the SV and right ventricular morphology, which are commonly referred to as single left ventricle (LV) and single right ventricle, respectively (Van Praagh et al., 1964; Anderson, 1984; Sherwood and Geva, 2002; Weinberg, 2002). However, these previous reports did not characterize the diagnostic imaging features and morphological characteristics of this condition in detail. Inaccurate diagnosis of SV and CoA might have important implications for catheter-based or surgical treatments. Prenatal echocardiography is a central component of monitoring and predicting fetus health, which is in turn dependent on a good understanding of fetal heart anatomy by clinicians for accurate, complete, and systematic evaluations. Digital visible human datasets is a new field emerging from the combination of information technology and medical courses (Robb, 2000). It integrates thousands of cross-sectional data of the human body and reconstructs a three-dimensional (3D) stereodisplay to provide a vivid model for human research. This subject has good prospects for applications in clinical imaging and anatomy. This study was undertaken to characterize the echocardiographic imaging, morphological, and myocardial pathological characteristics of SV connection with CoA, to reconstruct a 3-D digital visible heart model of the rare congenital heart disease in miscarried fetus cadavers, and to provide anatomical structures for constructing surgical and clinical image plans of the heart for medical teaching purposes.

\section{MATERIAL AND METHODS}

$\mathrm{SV}$ is a subclass of AV alignment abnormalities of double inlet and common inlet ventricles in which there is only one ventricular sinus (Van Praagh et al., 1979, 1982). Standard definitions of ventricular morphology were used to identify the right ventricle and the left ventricle (Van Praagh, 1984; Anderson, 1984; Van Praagh et al., 1989) of 15 specimens obtained from miscarried fetuses with the diagnosis of SV connected with CoA who had undergone prenatal echocardiograms at the Second Affiliated Hospital, Third Military Medical University since 2002 . 
Fourteen cardiac specimens were systematically reviewed for segmental anatomy, pulmonary venous connections, AV valve morphology, myocardial architecture, great vessel anomalies, and associated anomalies. All available postnatal echocardiograms were analyzed for the same anatomical details as in the specimen review. Prenatal echocardiograms were performed with an HP 5500 or a Philips iE33 cardiac imager equipped with a 3.5- or 5-MHz transducer. Conventional histological assays were performed on the 14 cases including myocardium of the apex of the heart and the vessel wall of the aorta in coarctation. Connective tissues were peeled from the heart and arterial walls. The specimens were fixed with $4 \%$ paraformaldehyde, embedded, sectioned, and stained with hematoxylin and eosin. Morphological changes were observed under light microscopy.

After 64-slice computed tomography angiography (CTA) and MRI were performed, 1 cadaver was embedded on $5 \%$ gelatin and cryopreserved at $-30^{\circ} \mathrm{C}$ for 2 weeks. A TK6350-digital milling machine (with milling accuracy of $0.001 \mathrm{~mm}$ ) was used to shave off slices of the fetal body layer by layer from head to foot at $-25^{\circ} \mathrm{C}$. The successive cross-sections were photographed with a high-definition digital camera. Thus, the structural dataset of the fetal body was completed. The area of the cardiac structural dataset of the fetus was chosen. Successive cross-sections were systematically reviewed for heart segmental anatomy, myocardial architecture, aortic and pulmonary outflow tract, great vessel anomalies, and associated anomalies. All available prenatal echocardiograms and CTAs were analyzed for the same anatomical details as in the dataset review. Standard definitions of ventricular morphology were used to identify the single LV. SV-complicated CoA was defined when there was only one ventricular sinus and when the aorta and the main pulmonary artery were aligned with the infundibulum. Transverse sectional anatomy data of the thoracic region, which included 232 consecutive slices, were chosen. The Adobe Photoshop software was used to segment the great vessels of the base of the heart, left and right atrium, ventricle, and descending aorta. Using the AMIRA software, the segmented structures were reconstructed in 3-D with volume- and surface-rendering reconstructions.

This study was carried out according to a specific protocol that was submitted to and approved by the Ethics Committees of the Second Affiliated Hospital, Third Military Medical University. Authorization for the scientific use of these materials was obtained from the parents.

\section{RESULTS}

The prenatal echocardiograms demonstrated SV and CoA in 15 cases. Thickening of the vessel wall in the ascending aorta was revealed (Figure 1A). The location of the coarctation area differed among cases including left anonyma, left common carotid artery, and left subclavian artery. Ultrasonic imaging also showed hypoplasia of the ascending aorta and aortic arch. The pulse Doppler ultrasound exhibited a "serrated" morphology in the ascending aorta spectra (Figure 1B).

Pathological dissections of specimens indicated hypertrophic myocardium in SV, significant aortic wall thickening, and localized coarctation area elevation, which resulted in obvious coarctation of the aorta (Figure 2a). Ten cases of SV with left ventricular morphology displayed a large muscle ridge (black asterisk, Figure 3A) and solitus normally aligned great arteries, i.e., the pulmonary artery arising from an anterior rightward right ventricular outlet chamber and aorta arising from the large LV. The left coronary artery was found to arise from 
the left side of the aortic root (Figure 3B). The muscle ridge can be mistaken for the ventricular septum by echocardiography. However, unlike a true ventricular septum, there is no characteristic LV septal surface. Furthermore, the muscle ridge was only present along the posterior wall of the ventricle and had no portion extending to the anterior or superior ventricular wall. Five cases of SV with right ventricular morphology showed coarse, parallel trabeculations (Figure $3 \mathrm{C}$ ) and received a common AV valve. The aorta was found to arise from the right ventricular cavity, and not from an outlet chamber.

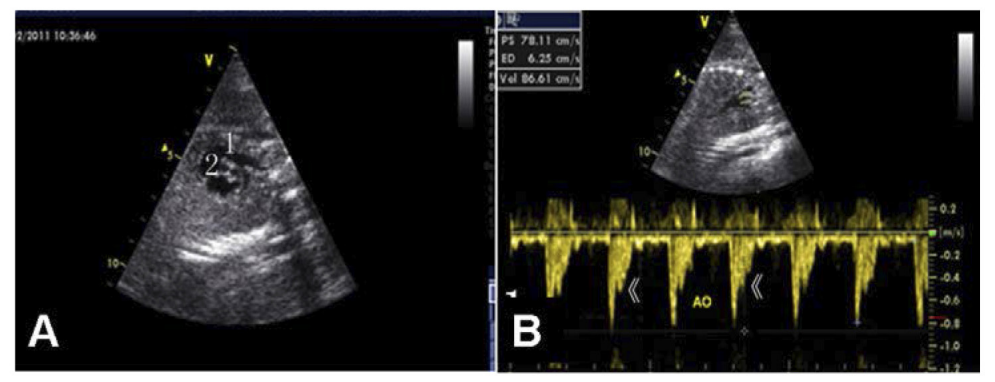

Figure 1. Prenatal echocardiography reveals thickening vessel wall in ascending aorta. A. Localized coarctation area elevation, which resulted in obviously coarctation of aorta. B. Pulse Doppler ultrasound exhibited a "serrated" morphology in ascending aorta spectra (arrows). $\mathrm{PA}=$ pulmonary artery; $\mathrm{AO}=$ aorta.

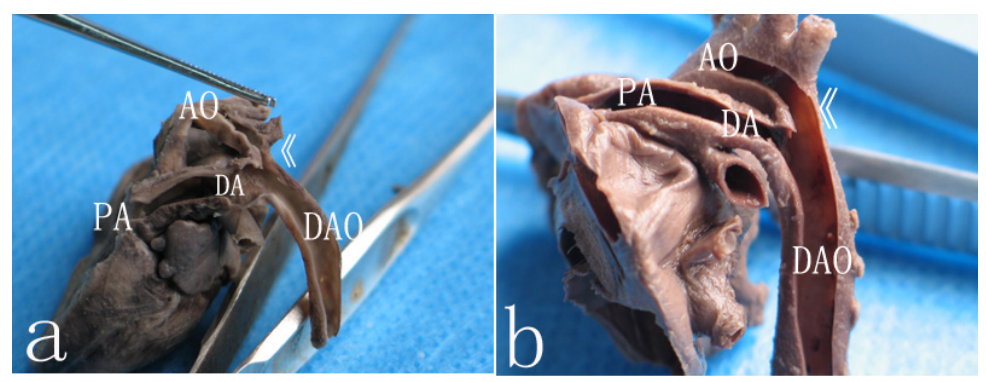

Figure 2. Pathological dissection of specimens. a. Significant aortic wall thickening and localized coarctation area (white arrows) elevation, which resulted in obvious coarctation of aorta. b. Normal structure of the aortic wall (white arrows). $\mathrm{AO}=$ ascending aorta; $\mathrm{PA}=$ pulmonary artery; $\mathrm{PDA}=$ ductus arteriosus; $\mathrm{DAO}=$ descending aorta.

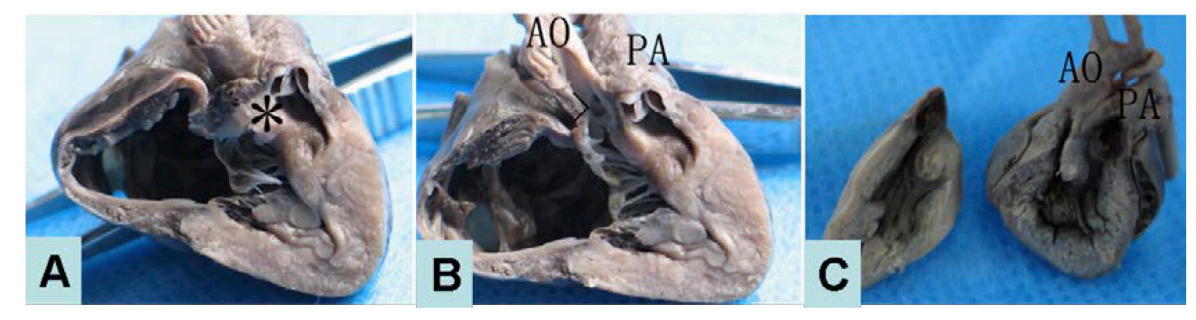

Figure 3. Case of single vanetricle (SV) with left ventricular morphology displays a large muscle ridge (asterisk, A), and solitus normally aligned great arteries, i.e., pulmonary artery (PA) arising from an anterior rightward right ventricular outlet chamber and aorta (AO) arising from the large left ventricle. The left coronary artery arises from the left side of the aortic root (B). Case of SV with right ventricular morphology has coarse, parallel trabeculations (C) and receives a common atrioventricular valve. The aorta is noted to arise from the right ventricular cavity. 
Light microscopy indicated hydropic degeneration, kytoplasm raritas and understain, muscle fiber prolongation, and thinning or twisting of cardiocytes. The interstitial tissues of the myocardium displayed hyperemia dropsy, a loose structure, and increased interspaces (Figure 4A). The thickness of the aortic wall was uneven. There were several red blood cells observed in the tunica adventitia of the artery. Hyperemia dropsy and loose structures were also displayed (Figure 5A).

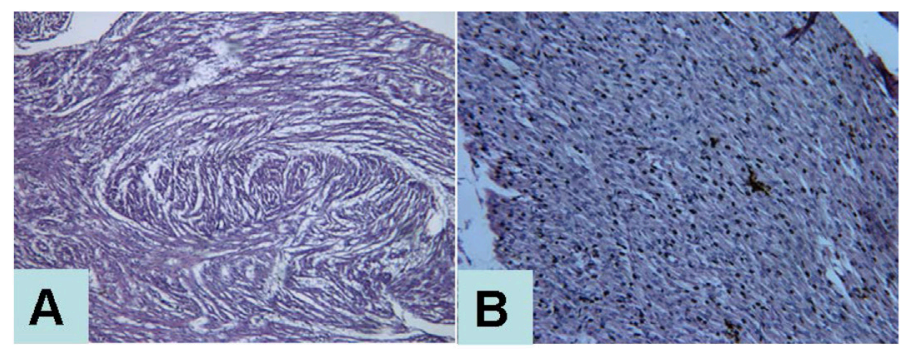

Figure 4. Light microscopy indicates hydropic degeneration, kytoplasm raritas and understain, muscle fiber prolong and thinning or twist in cardiocyte. Interstitial tissue of myocardium displays hyperemia dropsy loosing structure, and increasing interspace (A). B. Normal pathologic characteristical of myocardium.

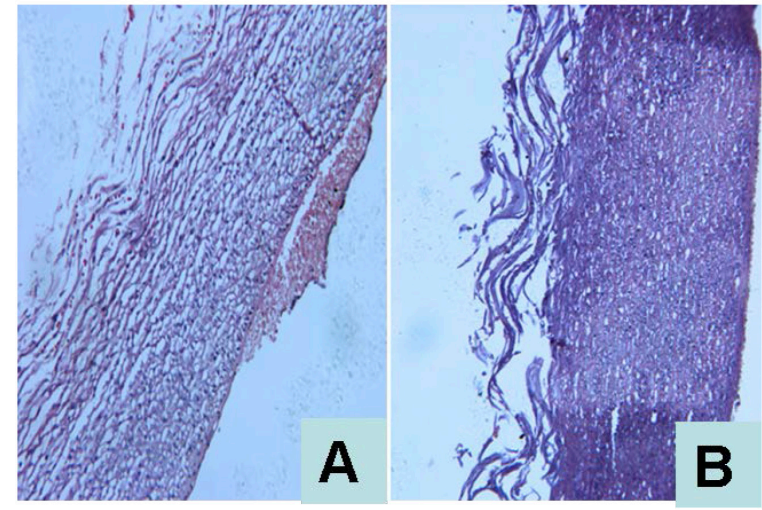

Figure 5. Light microscopy indicates that thickness was uneven in aortic wall. There are a number of red blood cells in the tunica adventitia of artery. Hyperemia dropsy and loosing structure are displayed (A). B. Normal pathological characteristics of aortic wall.

A specimen with a 35 -week gestation and a $2650 \mathrm{~g}$ weight was selected to reconstruct a 3-D digital visible heart model of SV connection with CoA. The imaging studies of the fetus were reviewed. Prenatal echocardiography revealed SV, aortic arch coarctation from the left common carotid artery, hypoplasia of the ascending aorta and aortic arch, atrial septal defect, gross pulmonary artery, and ductus arteriosus, through which the descending aorta linked to the pulmonary artery. Magnetic resonance image was performed with 3-mm thick specimens (Figure 6). CTA scans were conducted at $0.625-\mathrm{mm}$ intervals along the body, which clarified the anomalous heart anatomy as SV and CoA in the fetus cadaver (Figures 6, 7); however, this heart model had very low resolution and only a limited number of structures could be denoted. 
Later, accurate anatomical data became available. The thickness of each serial cross-section was $0.2 \mathrm{~mm}$ and 1495 slices were obtained. For each slice, each image was saved as a 31.4-MB file with a resolution of $10,989,056$ pixels ( $4064 \times 2704)$. The slices of the thoracic region were cut out of the original cross-sections and different tissues were segmented. The slices of the heart displayed a single large morphologically left ventricle. Significant myocardial hypertrophy was observed. Semi-automated segmentation and the Adobe Photoshop software were selected to segment the great vessels of the base of the heart, left and right atrium, ventricle, and descending aorta (Figures 8, 9). Figure 8A-C show the aortic arch coarctation. Figure 9A-C show the gross pulmonary artery and ductus arteriosus, through which the descending aorta linked to the pulmonary artery. All of the heart slices also had to be aligned to assure precise overlapping of the structures. Then, a 3-D reconstruction and stereodisplay of the fetus heart model with SV and CoA were established (Figure 10). Accurately segmented images of the main structures of the heart were completed. The reconstructed structures can be displayed singly, in small groups, or as a whole, and can be continuously rotated in 3-D space at different velocities.
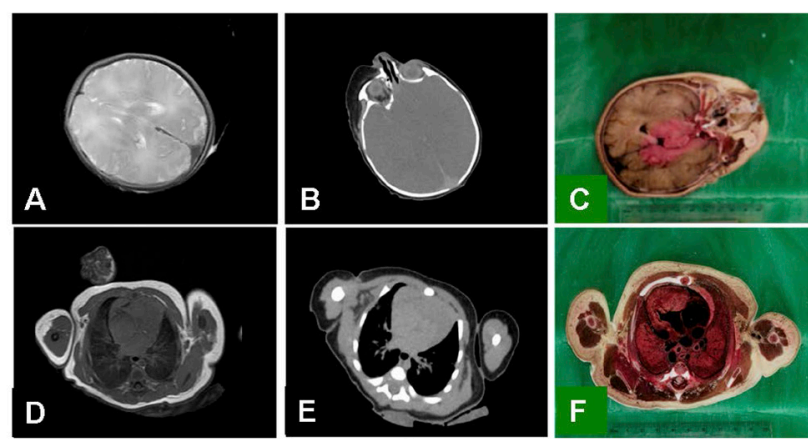

Figure 6. Slices of MRI, CT, and cross-sections of one cadaver. MRI with 3-mm thick slices for the body (A and D). CTA scans made in an interval of $0.625 \mathrm{~mm}$ for the body (B and E). Thickness is $0.2 \mathrm{~mm}$ for the serial crosssections $(\mathbf{C}$ and $\mathbf{F})$.
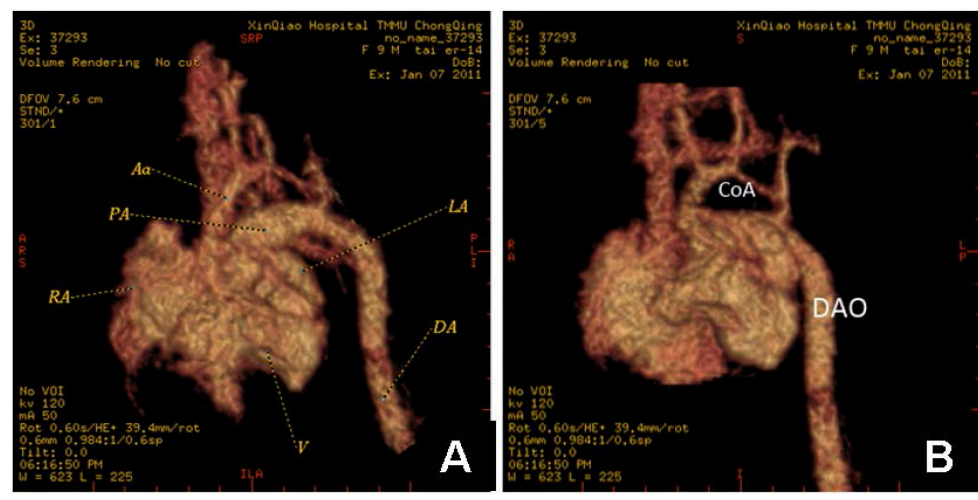

Figure 7. CTA scans of 64 slices clarify the anomalous heart anatomy as single ventricle (SV), hypoplasia of aortic arch, gross pulmonary artery (PA) and ductus arteriosus, through which the descending aorta (DAO) links to the PA (A), and aortic arch coarctation from left common carotid artery (B). V = single ventricle; Aa = ascending aorta; $\mathrm{DA}=$ descending aorta; $\mathrm{CoA}=$ aortic coarctation . 

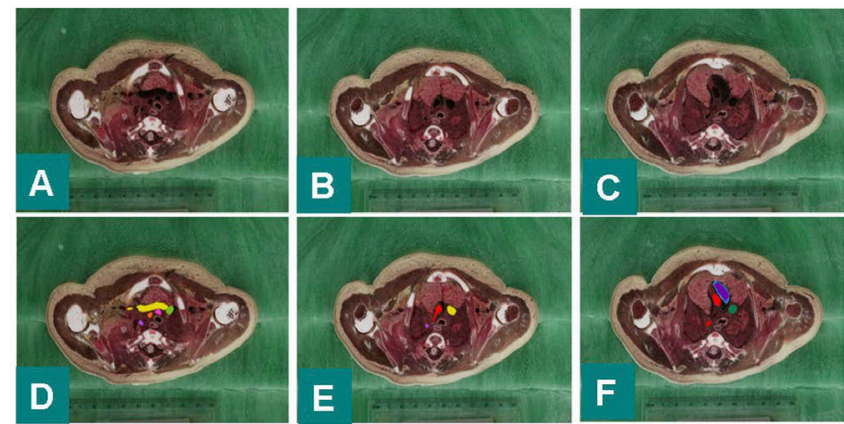

Figure 8. Cross-section image of the aortic arch reveals CoA; the thickness of the layer is $0.2 \mathrm{~mm}$ (A-C). Semiautomated segmentation and the Photoshop software were selected to segment great vessels on the base of the heart. The figure shows aortic arch, which are cropped and marked (D-F).
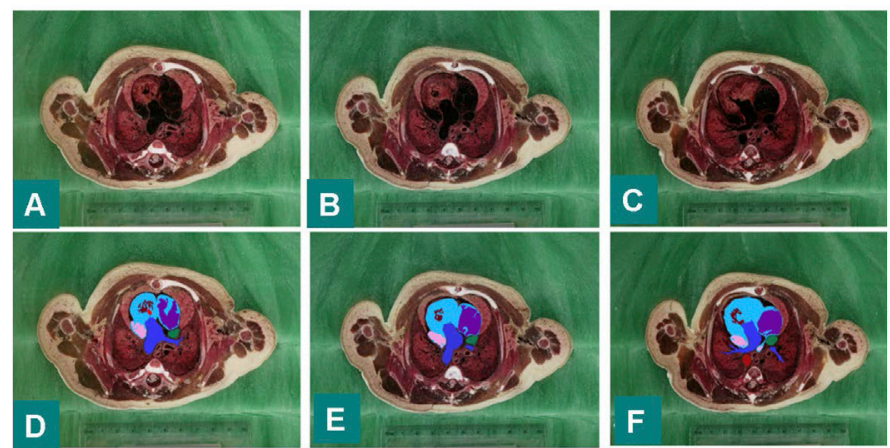

Figure 9. Section image of the pulmonary artery, left and right pulmonary arteries, ductus arteriosus, and descending aorta, where the thickness of the layer is $0.2 \mathrm{~mm}$, shows the gross pulmonary artery and ductus arteriosus, through which the descending aorta is linked to the pulmonary artery (A-C), which are cropped and marked (D-F).

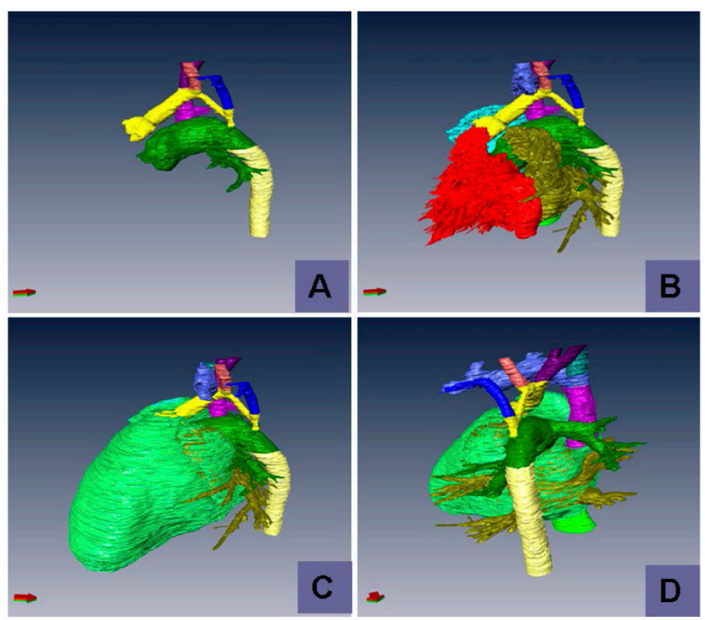

Figure 10. Reconstructed structures are displayed singly (A), in small groups (B) or as a whole and can be continuously rotated in 3-D spaces at different velocities $(\mathbf{C}$ and $\mathbf{D})$. 


\section{DISCUSSION}

Fetal autopsies are extremely important for accurate prediction of the recurrence risk of genetic conditions (Ramalho et al., 2006). Improving the diagnostic yield from post-mortem MR and CT imaging is currently an area of active research (Grabherr et al., 2008; Ross et al., 2008) with the aim of "Visible Humans" to have comparable accuracy to that of conventional autopsies. Several studies have been conducted in this field to date. Funded by the National Library of Medicine, the University of Colorado School of Medicine's Center for Human Simulation established the first visible human male dataset in 1995 (Spitzer et al., 1996; Spitzer and Whitlock, 1998). Each 24-bit true color image for a slice was saved as a 7.5MB file with a resolution of $2048 \times 1216$ pixels. The dissection was made at $1.0-\mathrm{mm}$ intervals for the whole body and 1871 slices were obtained. The whole data document reached 13 GB. In the Visible Human Project, the frozen corpse of a 59-year-old woman was serially sectioned (0.33-mm section cuts) and digitally imaged (Ackerman, 1991). From this dataset, a detailed 3 -D anatomical model of the whole human heart was constructed with a resolution of $330 \mu \mathrm{m}$ and 19 different tissue types (Boyett et al., 2005). In 2000, South Korea initiated a 5-year visible human dataset research project that aimed to build a dataset for the Asian population. The South Korean Ajou University established the first visible human dataset for an Asian adult of 210 GB in 2001. In 2002, the research group led by Professor S.X. Zhang consecutively completed data acquisition for the first visible human dataset for a Chinese male and female in 2002, making China the third country to complete a visible human dataset of its population (Li et al., 2006; Jin et al., 2008). Compared with the US and South Korea, the first Chinese visible human dataset is composed of successive $0.1 \mathrm{~mm}$-thick cross-sections, each reaching 6.3 million pixels. This technique reaches a world-class level owing to its data completeness, representativeness, and accuracy. The visible fetus dataset that we constructed in our study is the first of its kind in China.

Even though 3-D heart reconstructions have been previously reported in adult cadavers, there are no reports on fetal datasets, especially for fetuses with heart defects. In this study, our dataset was characterized by the following features. The selected specimen was a fetus with complicated heart abnormities, which will help research related to heart developmental defects. The serial cross-sections were $0.2 \mathrm{~mm}$ thick, thus ensuring complete and accurate data. Each slice image was saved as a 31.4-MB file with a resolution of 10,989,056 pixels (4064 x 2704). These high-definition digital images should prove useful for future research.

$\mathrm{SV}$ affiliated CoA is a type of complicated congenital heart disease. Accurate diagnosis using non-invasive imaging techniques is of paramount importance for optimal disease management. The reconstructed heart and the main internal structures are realistic, which demonstrates the natural shape and exact position of the structures. It provides an accurate model for automated segmentation algorithmic studies and a digital anatomical model of a fetus heart with SV and CoA.

Digital visible human dataset represents a technology that has limited applications for current teaching practices in medical universities of China, and is challenged by several hurdles. In research and applications of digital visible human datasets, image segmentation is required to expose human structures. The key technology of visualization of digital human heart datasets is segmentation. This technique delineates the target structures according to specific features, such as their gray scale, color, texture, and spectra. Image segmentation is 
a long-standing challenge from image treatment to analysis. Because segmentation can only make use of moiety features of the images, it is specific and limited in its application, which is selected based on actual needs. Regarding the large volume of data comprising the visible human dataset, how to treat the data is another concern accompanying accurate research interpretations and analyses. The large volume of data and complicated relationships between different structures are unprecedented. If the unit of the digital human model is cubic, the data increase by 8 -fold. Accordingly, if the thickness of the slice increases by 1 -fold, i.e., the data volume increases from 150 to $1200 \mathrm{~GB}$, the thickness changes from 0.2 to $0.1 \mathrm{~mm}$. The data may multiply if a 3-D reconstruction is enabled. Such a high volume of data poses a new challenge for data storage, transfer, and maintenance. How to compress these data without damage is a problem yet to be solved in establishing digital visible human datasets.

Prenatal ultrasonography is a useful and effective screening tool for fetus developmental defects owing to its non-invasiveness, replication, and reliability. Early intra-uterine therapy can be based on fetal developmental abnormalities as revealed by this examination. Advancing the digital visible dataset for fetuses is thus necessary to construct a digital 3-D fetus model. It will also update the teaching model for clinical imaging and medical teaching practices, increasing the interest of students, facilitating their logical thinking and communication, and enhancing broad teaching outcomes (Pflesser et al., 2001).

This study presented some initial attempts and results associated with a 3-D modeling of fetal hearts with SV and CoA. The basic data were taken from the fetal dataset. The resulting procedure can be used in some other areas of medicine, such as graphical presentations or as a learning tool for heart surgery.

In addition, previous studies identified a new molecular paradigm about NKx2-5 pathways for the onset of congenital heart defects and associated progressive cardiomyopathy in the steps that guide ventricular cells into discrete cardiomyocyte lineages (Pashmforoush et al., 2004; Chen et al., 2004). Since the ectopic ventricular expression of several genes is persistent and their effects are known to directly influence the automaticity, contractility, and trabeculation of cardiac muscles, our study about the pathological characteristics of the myocardial and vessel wall supports the concept that the surgical correction of structural morphogenic heart defects is unlikely to correct the underlying molecular defects, thereby leading to progressive dysfunction in the postnatal heart. It will be interesting to determine whether the antagonism of chronic gene signals might represent a new therapeutic approach to prevent progressive cardiac defects in genetic forms of human congenital heart disease.

\section{ACKNOWLEDGMENTS}

Research supported by the clinical research fund and the educational fund of the Third Military Medical University (\#2011XLC43 and \#2009B60), Chongqing (\#400038).

\section{REFERENCES}

Ackerman MJ (1991). The visible human project. J. Biocommun. 18: 14.

Anderson RH (1984). Univentricular hearts. Am. J. Cardiol. 54: 941-942.

Anderson RH, Becker AE, Freedom RM, Macartney FJ, et al. (1984). Sequential segmental analysis of congenital heart disease. Pediatr. Cardiol. 5: 281-287.

Boyett MR, Li J, Inada S, Dobrzynski H, et al. (2005). Imaging the heart: computer 3-dimensional anatomic models of the heart. J. Electrocardiol. 38: 113-120. 
Chen H, Shi S, Acosta L, Li W, et al. (2004). BMP10 is essential for maintaining cardiac growth during murine cardiogenesis. Development 131: 2219-2231.

Grabherr S, Gygax E, Sollberger B, Ross S, et al. (2008). Two-step postmortem angiography with a modified heart-lung machine: preliminary results. AJR Am. J. Roentgenol. 190: 345-351.

Jin Y, Chen G, Zhang SX, Tang LW, et al. (2008). Three-dimensional reconstruction of the pancreas and its surrounding structures. Comput. Med. Imaging Graph. 32: 277-283.

Li QY, Zhang SX, Heng PA, Liu ZJ, et al. (2006). Segmentation and three-dimension reconstruction of Chinese digitized human cerebrum. Comput. Med. Imaging Graph. 30: 89-94.

Pashmforoush M, Lu JT, Chen H, Amand TS, et al. (2004). Nkx2-5 pathways and congenital heart disease; loss of ventricular myocyte lineage specification leads to progressive cardiomyopathy and complete heart block. Cell 117: 373-386.

Pflesser B, Petersik A, Pommert A, Riemer M, et al. (2001). Exploring the visible human's inner organs with the VOXELMAN 3D navigator. Stud. Health Technol. Inform. 81: 379-385.

Ramalho C, Matias A, Brandao O and Montenegro N (2006). Critical evaluation of elective termination of pregnancy in a tertiary fetal medicine center during 43 months: correlation of prenatal diagnosis findings and postmortem examination. Prenat. Diagn. 26: 1084-1088.

Robb RA (2000). Virtual endoscopy: development and evaluation using the Visible Human datasets. Comput. Med. Imaging Graph. 24: 133-151.

Ross S, Spendlove D, Bolliger S, Christe A, et al. (2008). Postmortem whole-body CT angiography: evaluation of two contrast media solutions. AJR Am. J. Roentgenol. 190: 1380-1389.

Sherwood MC and Geva T (2002). Noninvasive imaging of the single ventricle. Progr. Pediatr. Cardiol. 16: 11-30.

Spitzer V, Ackerman MJ, Scherzinger AL and Whitlock D (1996). The visible human male: a technical report. J. Am. Med. Inform. Assoc. 3: 118-130.

Spitzer VM and Whitlock DG (1998). The Visible Human Dataset: the anatomical platform for human simulation. Anat. Rec. 253: 49-57.

Van Praagh R (1984). The segmental approach clarified. Cardiovasc. Intervent. Radiol. 7: 320-325.

Van Praagh R, Ongley PA and Swan HJ (1964). Anatomic types of single or common ventricle in man. morphologic and geometric aspects of 60 necropsied cases. Am. J. Cardiol. 13: 367-386.

Van Praagh R, Plett JA and Van Praagh S (1979). Single ventricle. Pathology, embryology, terminology and classification. Herz 4: 113-150.

Van Praagh R, David I and Van Praagh S (1982). What is a ventricle? The single-ventricle trap. Pediatr. Cardiol. 2: 79-84.

Van Praagh R, Geva T and Kreutzer J (1989). Ventricular septal defects: how shall we describe, name and classify them? J. Am. Coll. Cardiol. 14: 1298-1299.

Weinberg PM (2002). Morphology of single ventricle. Progr. Pediatr. Cardiol. 16: 1-9. 